Wolling, Jens; Schmolinsky, Anja; Emmer, Martin:

Politiker vernetzt: wie und warum sich Landtagsabgeordnete online präsentieren

Original erschienen in:

Politik 2.0? : die Wirkung computervermittelter Kommunikation auf den politischen Prozess. - Baden-Baden : Nomos, Ed. Fischer, 2010, S. 59-83.

(Internet research ; 38)

ISBN (print): 978-3-8329-5300-3

ISBN (online): 978-3-8452-2346-9

ISSN (print): $1617-6839$

DOI: $\quad 10.5771 / 9783845223469-59$

URL: $\quad$ http://www.nomos-elibrary.de/10.5771/9783845223469-59/

[Gesehen: 01.02.2016] 


\section{Politiker vernetzt: Wie und warum sich Landtagsabge- ordnete online präsentieren}

„Ein Politiker, der heute keine Internetpräsenz beweist, hat meines Erachtens die Zeichen der Zeit nicht verstanden. Mebr noch: In meinen Augen nimmt er seine Aufgaben und Pflichten nicht vollständig wabr, wenn die Menschen keine Gelegenbeiten baben sich über seine Tätigkeit zu informieren."

„Das Internet ist für mich ein weiteres Element geworden, um politische Rückmeldung zu bekommen. Zu meinen Aufgaben gebört auch die Organisation direkter politischer Kommunikation. Die Interaktion über das Netz ist dabei ein weiteres Standbein geworden. Deshalb ist es für mich auch selbstverständlich, mehrmals am Tag meine Mails selbst zu lesen und zu bearbeiten. Kein Büro und kein Referent sind dazwischen geschaltet."

Mit diesen geradezu euphorischen Worten beschreiben eine Abgeordnete des Europaparlaments (Koch-Mehrin, 2006) und ein Landtagsabgeordneter aus Hessen (Siebel, 2006) die Funktion der Onlinepräsenz für ihre politische Arbeit. Das Internet ist für sie nicht nur ein Instrument, um die Wähler über das eigene politische Handeln zu informieren, sondern bietet auch die Möglichkeit, die Bürger zu Stellungnahmen und zur Diskussion zu ermuntern, um auf diese Weise Anstöße für die weitere politische Arbeit zu bekommen.

Diese Einschätzung scheinen mittlerweile die meisten Abgeordneten zu teilen, denn es gibt kaum noch Politiker, die meinen, auf politische Internetaktivitäten verzichten zu können. Schon Mitte der 1990er Jahre entstanden die ersten individuellen Internetseiten, auf denen sich Politiker der Öffentlichkeit präsentierten (Zittel, 2005: 8f.). 1998 hatten bereits 30 Prozent der Bundestagsabgeordneten eine persönliche Website (Döring, 2003); im Jahr 2006 war der Anteil auf 98 Prozent gestiegen (Rolke/Metz, 2006). Unter den Landtagsabgeordneten verlief diese Entwicklung nicht ganz so schnell: Im Jahr 2002 belief sich der Anteil der Abgeordneten mit eigener Website auf 31 Prozent (Döring, 2003). Zwei Jahre später waren es knapp die Hälfte und 2007 immerhin schon 68 Prozent (Altmeyer et al., 2007).

Warum Politiker ihrer Internetpräsenz eine zunehmende Beachtung schenken, ist nicht zuletzt auf die wachsende Reichweite von Politiker- und Parteiwebsites generell zurückzuführen. Mittlerweile gehört jeder sechste Bundesbürger zum Nutzerkreis (Emmer/Wolling, 2009) solcher Angebote. Andererseits zeigen inhaltsanalytische Untersuchungen (Druckman et al., 2007; Schweitzer, 2005), dass Internetseiten von Politikern und Parteien die Möglichkeiten dieser Kommunikationsform bisher nur partiell ausschöpfen. Vor diesem Hintergrund stellt sich die Frage, welche konkreten Zielset- 
zungen, Ansprüche und Erwartungen Politiker mit ihren Websites verbinden. Sind die eingangs zitierten Vorstellungen repräsentativ für die Abgeordneten oder handelt es sich dabei doch um eher untypische Ideen einzelner Internetaktivisten? Besondere Aufmerksamkeit verdienen in diesem Zusammenhang die Konsequenzen eben jener Vorstellungen: Beeinflussen sie die Auswahl der individuellen Maßnahmen zur Ausgestaltung der persönlichen Website oder werden diese Entscheidungen doch vor allem durch die strukturellen Rahmenbedingungen bestimmt, die zeitlichen Restriktionen, die Kompetenzen oder die finanziellen Ressourcen? Diese Fragen sollen im vorliegenden Beitrag untersucht werden. Ziel ist es, ein theoretisch fundiertes Erklärungsmodell für die Unterhaltung privater Websites vorzulegen und dies am Beispiel deutscher Landtagsabgeordneter empirisch zu prüfen.

\section{Forschungsstand: Online-Präsenzen von Politikern}

Dem Untersuchungsgegenstand „Politikerwebsites“ kann man sich von unterschiedlichen Seiten nähern. Aus der Nutzerperspektive interessiert zum einen, wie intensiv und von welchen Bevölkerungsgruppen die verschiedenen Angebote genutzt werden. Hierzu liegen Daten einer repräsentativen Langfriststudie vor, die belegt, dass das Interesse an den Seiten kontinuierlich gewachsen ist (Emmer/Wolling, 2009). Bereits Anfang des Jahrzehnts besuchte ein Viertel bis ein Drittel der damals allerdings noch relativ kleinen Internetgemeinde solche Angebote (Emmer, 2005: 124). Mittlerweile gehört jeder sechste Bundesbürger zum Nutzerkreis. In einzelnen Bevölkerungssegmenten ist der Anteil sogar noch deutlich höher. Insbesondere unter den politisch Interessierten $(26 \%)$ und den formal höher Gebildeten (30\%) finden sich viele Personen, die Politikerwebsites aufsuchen (Emmer/Wolling, 2009).

Aber nicht nur die Nutzungszahlen sind von Interesse, sondern auch die Qualitätserwartungen der Rezipienten (und potentiellen Wähler), denn sie sind neben Journalisten (als potentielle Multiplikatoren) eine wichtige Zielgruppe der Websites. Welche Elemente und Angebotsformen eine gute Internetseite aus der Sicht der Nutzer haben sollte, wurde in einer Befragung für das Projekt „eCandidates 2002“ (eCandidates 2002, 2002) untersucht. Ein wichtiges Ergebnis dieser Studie war die Ermittlung von Inhalten und Gestaltungselementen, die nach Einschätzung der Bürger auf einer Politikerwebsite vorhanden sein sollten. Des Weiteren stellte sich heraus, dass die Hälfte der befragten Onliner die Internetpräsenz von Politikern als wichtig oder sogar sehr wichtig einschätzte.

Eine zweite Perspektive auf das Phänomen ist die Analyse der Inhalte. Schon frühzeitig wurden Inhaltsanalysen von Partei- und Politikerwebsites durchgeführt. Neben theoriebasierten und wissenschaftlich orientierten Analysen wurden hierzu - vor allem vor 
Wahlen - auch praxisorientierte „Webseitentests“ von Politikberatungsorganisationen und politischen Fachpublikationen durchgeführi (etwa von politik-digital.de oder „Politik \& Kommunikation“). Analysiert wurde dabei meist die kommunikative Qualität der Angebote, der Grad der Interaktivität, die Usability etc.

Paschen und Wieboldt (2001) stellten bei einer Analyse der Websites von Bundestagsabgeordneten 2001 fest, dass sich gegenüber einer früheren Analyse aus dem Jahr 1998 zwar die Aktualität verbessert und der Einsatz interaktiver Elemente zugenommen hat, bemängelten aber, dass sich beides noch immer nicht auf einem zufriedenstellenden Niveau befindet. Die Studie „eCandidates 2002“ untersuchte neben Nutzererwartungen (s. o.) im Vorfeld der Bundestagswahl auch inhaltsanalytisch die Webpräsenzen der Kandidaten für den Deutschen Bundestag. Hauptkritikpunkt war auch hier die fehlende Aktualität der Angebote. Döring (2003) führte eine Inhaltsanalyse von Gästebucheinträgen durch und ermittelte die Responsivität der Politiker auf E-MailAnfragen. Ihre Ergebnisse zeigen, dass es sich bei der Hälfte der Gästebucheinträge um Unterstützungsbekundungen für den Politiker handelt, 20 Prozent beinhalten Kritik, 15 Prozent sind Hilfegesuche und weitere 15 Prozent sind unpolitisch. Die Responsivität auf E-Mail-Anfragen betrug innerhalb einer Woche gerade einmal 28 Prozent. Ähnliche Ergebnisse zeigen Analysen von Partei-Websites wie z. B. von Schweitzer (2005): Wieder findet sich insgesamt wenig Interaktivität, inhaltliche Unterschiede zwischen Parteien sind geringer als erwartet. Zittel (2003) führte im Jahr 2000 eine international vergleichende Inhaltsanalyse von Abgeordnetenwebsites durch. Es wurde deutlich, dass die amerikanischen Abgeordneten des Repräsentantenhauses fast alle über eine eigene Website verfügen, während es bei den deutschen Bundestags- und den schwedischen Riksdag-Abgeordneten nur rund ein Drittel ist. Übereinstimmend konnte aber für alle drei Länder ermittelt werden, dass die anspruchsvolleren interaktiven Anwendungen (jenseits von E-Mails) kaum genutzt werden. Die Hauptzielgruppe der Websites waren in allen drei Ländern nicht die Bürger, sondern die Medien. Zwei Jahre später präsentierte Zittel (2005) eine vergleichende Inhaltsanalyse aller Websites der Bundestagsabgeordneten aus den Jahren 2000 und 2004. Er konnte dabei unter anderem feststellen, dass die Verbreitung zahlreicher Angebotsformen wie Positionspapiere, Presseerklärungen, Newsletter und interaktive Kommunikationsmodi im Untersuchungszeitraum zugenommen hat. Wiederum zwei Jahre später dehnte er diesen zeitlichen Vergleich auch auf Schweden und die Vereinigten Staaten aus (Zittel, 2007). Seine Vermutung, dass die Entscheidung für eine Onlinepräsenz durch wahlsystemische Anreize (z. B. Direkt- vs. Listenwahl) beeinflusst wird, konnte er im zweiten Zeitraum nicht bestätigen. Ebenfalls im Jahr 2007 haben Altmeyer et al. (2007) einen Test der Websites von 2456 Abgeordneten aus Bund und Ländern durchgeführt und dazu 
Rankings vorgelegt. Im Unterschied zu früheren Studien konnten sie einen hohen Aktualisierungsrhythmus feststellen.

Ein dritter Zugang, der auch für die hier vorgelegte Untersuchung gewählt wurde, ist die Befragung der Abgeordneten selbst. Untersuchungen der Kommunikatoren, ihrer Motive und Ziele bei der Einrichtung und Gestaltung ihrer Online-Präsenzen liegen bisher nur fragmentarisch vor. Gelegentlich wurde im Rahmen umfassender Studien zur Kommunikation von Politikern auch nach der Bedeutung des Internets gefragt. Kleinsteuber und Fries (2003) beispielsweise haben bei einer schriftlichen Befragung der Bundestagsabgeordneten herausgefunden, dass die Nutzung des Internets zumeist auf die Eigeninitiative der Abgeordneten zurückzuführen war. Interaktive Angebote wie Newsgroups, Diskussionsforen oder Chats wurden von ihnen nur selten genutzt. Aber immerhin rund ein Drittel der Befragungsteilnehmer gab an, dass E-Mails für ihre politische Arbeit wichtig seien. Im gleichen Jahr hat Faas (2003) eine Befragung von Landtagsabgeordneten aus den Bundesländern Baden-Württemberg, Rheinland-Pfalz und Sachsen-Anhalt veröffentlicht. Die Ergebnisse seiner Studie zeigten, dass das wichtigste Motiv zur Einrichtung einer Website die Selbstdarstellung ist. Als weitere Motive identifizierte er den Wunsch, Kontaktmöglichkeiten zu schaffen und bestimmte Wählergruppen zu erreichen. Als Hindernisse für die Einrichtung einer Website wurden geringe zeitliche und finanzielle Ressourcen sowie fehlende Internetkompetenz genannt. Rolke und Metz (Rolke/Metz, 2006) haben eine Intensivbefragung von 119 Bundestagsabgeordneten durchgeführt: Die Abgeordneten nannten Stamm- und Wechselwähler als wichtigste Zielgruppen ihrer Websites, danach folgten Journalisten, Parteimitglieder und Mitarbeiter. Zittel untersuchte Politikerwebsites nicht nur inhaltsanalytisch (s. o.), sondern hat auch mehrfach $(2005 ; 2007$; 2009) Ergebnisse qualitativer Leitfadeninterviews mit Abgeordneten veröffentlicht, in denen er deren Erwartungen an die Funktion von privaten Websites sowie deren Beurteilung und Nutzung ermittelt hatte. In der jüngsten Veröffentlichung - in der er auf die Interviews mit Bundestagsabgeordneten aus dem Jahr 2004 zurückgreift - stellte er fest, dass die Abgeordneten neben den Wählern auch die Fachöffentlichkeit bestimmter Verbände erreichen wollen. Ihre Webpräsenz ist für sie einerseits ein Mittel, um die Massenmedien bei der Zielgruppenansprache zu umgehen, andererseits sind sie der Auffassung, dass eine Website die Präsenz in den Massenmedien nicht ersetzen kann. Die befragten Mitglieder des Bundestages bezweifelten, dass die Website einen wahlentscheidenden Einfluss hat, aber nutzten sie trotzdem für Wahlwerbung und vertraten zudem die Ansicht, dass man mit einer Website eine Wahl zwar nicht gewinnen, aber obne eine Website durchaus verlieren könne. Hinsichtlich der Funktion interaktiver Angebote zeigten sich die Abgeordneten eher skeptisch. Dabei wurden auch strategische Überlegungen deutlich. Die Abgeordneten machten generell die Nutzungsentscheidungen abhängig von den Erwartungen 
der Wählerschaft und damit von der Struktur ihres Wahlkreises sowie im besonderen Maße auch von den Erwartungen parteiinterner Zielgruppen, die bei der Aufstellung von Kandidatenlisten von Bedeutung sind. Aufgrund solcher komplexer strategischer Überlegungen erwiesen sich nach Ansicht von Zittel (2009) auch einfache Unterscheidungen zwischen Direkt- und Listenkandidaten für die Ausgestaltung der Seiten als wenig relevant.

\section{Theoretische Grundlagen: Motive und Erwartungen}

In der Literatur zur politischen Funktion des Internets wird immer wieder dessen interaktives Potenzial hervorgehoben (Beck, 2006; Rössler, 1998). Demnach bietet das Internet den Abgeordneten die Chance, in direkten Austausch mit den Bürgern zu treten und so den demokratischen Prozess der Willensbildung zu stärken. Vor diesem Hintergrund kann es kaum überraschen, dass die Interaktivität der Online-Angebote in praktisch allen oben vorgestellten Studien thematisiert wird. Immer wieder zeigte sich, dass anspruchsvollere Formen des politischen Austauschs in den Angeboten der Politiker nur eine geringe Rolle spielen. Andererseits ist aber auch bekannt, dass viele Bürger solche Möglichkeiten des Austauschs zwar fordern, jedoch nur wenige diese Optionen auch tatsächlich nutzen (Emmer/Wolling, 2009). Die Zurückhaltung ist möglicherweise auch darauf zurückzuführen, dass Frustrationen bei diesen Kommunikationsformen vorprogrammiert sind. Hierfür gibt es mehrere denkbare Gründe: Zum einen ist es für die Abgeordneten schon aus Zeitgründen schwierig, mit einer Vielzahl von Bürgern einen intensiven Austausch zu pflegen; zum anderen kann dies aber auch darauf zurückzuführen sein, dass sie dieser Form des Austauschs keine besonders große Bedeutung zumessen. Für die Abgeordneten ist es zwar wichtig, die Wünsche und Erwartungen der Bürger zu kennen, um auf diese reagieren zu können, da ein Mindestmaß an Responsivität für den Erhalt der eigenen Wahlchancen unerlässlich ist, allerdings stellt sich die Frage, ob die Rückmeldungen einzelner aktiver Bürger einen repräsentativen Indikator für die Ansichten des Elektorats darstellen. Wenn man von der Annahme ausgeht, dass Abgeordnete versuchen (müssen), ihre Ressourcen effektiv einzusetzen, dann ist zu erwarten, dass sie diesen anspruchsvolleren Angebotsformen bei knappen Ressourcen eher geringe Aufmerksamkeit schenken und sich stattdessen auf traditionellere Formen der Informationsvermittlung und Selbstdarstellung konzentrieren.

Indes erscheint die Fokussierung der Forschung auf interaktive Angebote etwas einseitig und dem komplexen Gegenstand nicht angemessen, denn ein substanzieller Beitrag zu mehr Transparenz und Qualität des politischen Prozesses kann sicher auch durch andere Eigenschaften von Webangeboten geleistet werden: etwa durch die Bereitstellung detaillierter politischer Informationen, die Offenlegung politischer Zielset- 
zungen oder die umfangreichere Darstellung der Persönlichkeit des Politikers. Somit erscheint es auch aus normativer Sicht angebracht, sich in der vorliegenden Studie nicht nur auf die Untersuchung der Interaktivitätsdimension zu beschränken.

Theoretisch basiert die Untersuchung auf der Annahme, dass sich Abgeordnete aus rationalen Überlegungen für (oder gegen) die Einrichtung und die spezifische Ausgestaltung einer privaten Website entscheiden. Sie wägen ab zwischen den vermuteten Nutzen, die ihnen die Installation der Seite und die Implementation verschiedener Angebote auf dieser Seite bringen wird, und den Kosten, die durch die Einrichtung und die kontinuierliche Pflege entstehen. Die Forschung bewegt sich also in der Tradition rationaler Handlungstheorien, deren Grundüberlegungen aus der Ökonomie stammen (Eisenführ/Weber, 2003), mittlerweile jedoch in verschiedenen anderen Wissenschaften genutzt werden (Esser, 1999), unter anderem in der Politikwissenschaft - beispielsweise bei der Erklärung des Wahlverhaltens (Kühnel, 2001; Shahla, 1999; Thurner/Roth, 2001).

Die Grundideen der Theorie des rationalen Entscheidens sind auch in der Kommunikationswissenschaft in unterschiedlichen Forschungsfeldern aufgegriffen worden. Insbesondere in der Rezeptionsforschung spielen diese Vorstellungen in den verschiedenen Varianten des Uses-and-Gratifications-Ansatzes eine große Rolle. Im Rahmen dieser Forschungstradition steht die Untersuchung der handlungsleitenden Motive der Rezipienten im Mittelpunkt. Hierzu liegen eine Vielzahl von Studien zu den unterschiedlichsten Medien vor, die die jeweiligen Nutzungsentscheidungen der Rezipienten aus deren Motiven heraus zu erklären versuchen (im Überblick Schweiger, 2007). Speziell für die Erklärung der Internetnutzung bietet sich der Ansatz an, da hier die Selektivität und damit aktive Nutzungsentscheidungen eine wesentlich größere Rolle spielen als bei anderen Medien (Papacharissi/Rubin, 2000).

Die Übertragung des Menschenbildes des Homo Oeconomicus und der Grundideen des Uses-and-Gratifications-Ansatzes in die Kommunikatorforschung erscheint vor diesem Hintergrund nur folgerichtig und wurde bereits realisiert (Fengler/RußMohl, 2005). Die zunehmende Vermischung von Produzenten- und Rezipientenrollen im Internet ist ein weiteres Argument, das die Anwendung des theoretischen Ansatzes bei der Erforschung der Internetnutzung von Abgeordneten angemessen erscheinen lässt. Die vorliegende Untersuchung fragt deshalb auf Basis des Uses-andGratifications-Ansatzes nach konkreten Motiven von gewählten Abgeordneten, eine Internetpräsenz einzurichten und bestimmte Kommunikationsangebote auf dieser anzubieten oder darauf zu verzichten.

Um herauszufinden, welche Vorteile die Abgeordneten von einer Website erwarten, müssen somit ihre Nutzungsmotive erforscht werden. Dem Uses-and-GratificationsAnsatz wurde vielfach Theorieschwäche oder sogar Theorielosigkeit vorgeworfen (vgl. 
im Überblick zu den Kritikpunkten am Uses-and-Gratifications-Ansatz Schweiger, 2007). Als Reaktion auf diese Kritikpunkte wurden die relativ einfachen Ausgangsüberlegungen sowohl theoretisch als auch in der Forschungspraxis weiterentwickelt. Eine dieser Weiterentwicklung war die Unterscheidung von gesuchten und erhaltenen Gratifikationen (Palmgreen et al., 1980; Wenner, 1982) sowie die theoretische Anbindung an das Wert-Erwartungsmodell (Esser, 1999; Rayburn/Palmgreen, 1984). Beide Weiterentwicklungen werden im Rahmen dieser Studie berücksichtigt.

Die Motive sind die Antriebskräfte, die die Abgeordneten veranlassen, bestimmte kommunikative Maßnahmen zu ergreifen. Aus der Perspektive der Theorie der rationalen Entscheidung sind jedoch die situationellen Bedingungen (Esser, 1999) für die individuellen Handlungsentscheidungen von noch größerer Bedeutung als die Motive, denn Handlungen werden stärker von den damit verbundenen Kosten beeinflusst als von den Wünschen der Handelnden. Gerade bei der Internetnutzung können Optionen und Restriktionen einen erheblichen Einfluss auf die Nutzungsentscheidungen haben (Vowe/Wolling, 2002). Optionen und Restriktionen sind zwei Seiten der gleichen Medaille: So eröffnet beispielsweise die hohe Internetkompetenz seiner Mitarbeiter einem Abgeordneten die Option, eine anspruchsvolle Website zu installieren; fehlende Kompetenzen wirken sich als Restriktion eher hemmend aus. Neben den Kompetenzen sind vor allem zeitliche und finanzielle Ressourcen von Bedeutung. Zittel (2009) hat verschiedentlich darauf hingewiesen, dass auch Besonderheiten des Wahlsystems einen Einfluss auf die Nutzung von Abgeordnetenwebsites haben können. Seine Forschungsergebnisse verdeutlichen, dass die Zusammenhänge sich hier komplizierter darstellen, als es auf den ersten Blick scheint. Aus diesem Grund sollte der mögliche wahlsystemische Einfluss nicht nur - wie in früheren Studien - auf die Einrichtung einer Website, sondern auch auf deren Ausgestaltung untersucht werden.

Der dritte Faktor, der im Erklärungsmodell Berücksichtigung finden muss, sind die sozialen Einflüsse. Theoretisch lassen sich soziale Einflüsse unterschiedlich interpretieren. So können sie - wie in der Schweigespirale (Noelle-Neumann, 1982) - als Reaktionen auf Isolationsfurcht begriffen werden. Folgt man dieser Auffassung, dann beobachten die Abgeordneten ihre Umwelt, um die Erwartungen der relevanten Bezugsgruppen zu erkennen. Wenn sie zu der Ansicht gelangen, dass die relevanten Anderen die Einrichtung und spezifische Ausgestaltung einer Website für normativ geboten halten (eine solche normative Begründung wurde in der eingangs zitierten Aussage von Koch-Mehrin deutlich), dann werden sie dazu neigen, diese Erwartungen zu erfüllen. Die Wirkung des sozialen Einflusses lässt sich aber auch in der Logik der Theorie der rationalen Entscheidung erklären. Die Orientierung an relevanten Bezugsgruppen kann einerseits durch das Bedürfnis nach sozialer Wertschätzung (Esser, 1999: 125f.) erklärt werden, anderseits gehören aber die sozialen Beziehungen zu den situativen Bedingun- 
gen, die von den Handelnden berücksichtigt werden müssen, wenn sie ihre Ziele erreichen wollen. Aus dieser Perspektive ist es rational zu berücksichtigen, was relevante Bezugsgruppen erwarten.

\section{Forschungsfragen und methodisches Vorgehen}

Aus den im Forschungsüberblick genannten inhaltsanalytischen Studien ist bereits einiges über die Ausgestaltung der privaten Websites ${ }^{1}$ der Abgeordneten bekannt. Aus den Befragungen und Interviews kennen wir Präferenzen und einzelne Motive für die Nutzungsentscheidungen. Auch verschiedene Restriktionen sind in den Studien diskutiert worden. Welchen Beitrag diese verschiedenen Faktoren dabei leisten können, die Entscheidung für die Nutzung bestimmter Angebotsoptionen zu erläutern, ist aber noch weitgehend ungeklärt. Im Theorieteil wurden die individuellen Motive, die strukturellen Rahmenbedingungen sowie soziale Einflüsse als mögliche Erklärungsfaktoren für diese Entscheidungen diskutiert. Das Ziel der nachfolgend vorgestellten empirischen Untersuchung ist es, die Relevanz der Faktoren zu bestimmen, um nachfolgende Forschungsfrage zu beantworten:

FF: Durch welche Faktoren werden die Entscheidungen für spezifische Ausgestaltungsoptionen der persönlichen Website beeinflusst?

Als Zwischenschritte zur Beantwortung dieser forschungsleitenden Frage sollen zuvor zwei weitere deskriptive Fragen beantwortet werden:

Frage 1: Welche Landespolitiker nutzen eine persönliche Website für ihre politische Kommunikation und wie intensiv pflegen sie diese?

Frage 2: Für wie bedeutsam erachten die Abgeordneten die unterschiedlichen Ausgestaltungsoptionen für ihre persönliche Website?

\section{Grundgesamtheit und Stichprobe}

Die Datengrundlage der Untersuchung bildet eine repräsentative Befragung von Landtagsabgeordneten. Die Grundgesamtheit dieser Befragung bilden die Mitglieder der 16 bundesdeutschen Länderparlamente, die einer der nachfolgenden sechs Fraktionen an-

1 Mit „privaten Websites“ sind hier und im weiteren Verlauf der Untersuchung solche Webangebote gemeint, die von den Abgeordneten selbst (bzw. in ihrem Auftrag) betrieben und finanziert werden (und die in der Regel über Domainnamen, die den Namen der Person enthalten, erreichbar sind). Nicht Gegenstand der Untersuchung sind personenbezogene Seiten, die dem Webangebot von Parlamenten oder Parteien untergeordnet sind und die in der Regel von diesen Organisationen erstellt und zentral über Partei- oder Parlamentsressourcen gepflegt werden. 
gehören: CDU, CSU, SPD, Bündnis 90/Die Grünen, FDP, Die Linke. Zum Befragungszeitraum waren dies insgesamt 1814 Personen. Daraus wurde eine disproportional geschichtete Stichprobe gezogen. Schichtungskriterien waren Bundesland und Fraktion. Kleinere Parlamente und Fraktionen wurden überproportional berücksichtigt. Während aus den Fraktionen der CDU bzw. CSU und der SPD jeweils 165 Abgeordnete per Zufall in die Stichprobe gewählt wurden, waren es bei FDP, Bündnis 90/Die Grünen und Die Linke jeweils 80. Aus Länderparlamenten mit über 140 Abgeordneten wurden jeweils 40, aus denen mit 80 bis 140 wurden 35 und aus denen mit unter 80 Abgeordneten wurden je 30 in die Stichprobe gezogen. Für die folgenden Datenauswertungen wurden die durch das Auswahlverfahren entstandenen Verzerrungen durch Datengewichtung wieder ausgeglichen.

Die so ausgewählten 570 Landtagsabgeordneten wurden im Oktober 2007 postalisch kontaktiert und um Kooperation gebeten. Die im Anschreiben mit gesendeten Fragebögen konnten sowohl auf dem Postweg als auch per Fax zurückgeschickt werden. Darüber hinaus bestand die Möglichkeit, den Fragebogen online auszufüllen. Die Rücklaufquote von 26 Prozent $(n=150)$ liefert eine qualitativ hochwertige Datenbasis. Nur selten werden ähnlich gute Ergebnisse bei Politikerbefragungen erreicht (Marx, 2009: 71). Häufig liegen die Quoten deutlich unter 20 Prozent (Kleinsteuber/Fries, 2003: 93). Die relative hohe Teilnahmebereitschaft könnte unter anderem darauf zurückzuführen sein, dass es den Abgeordneten explizit freigestellt wurde, den Fragebogen selbst zu beantworten oder dies an den für das Online-Angebot verantwortlichen Mitarbeiter zu delegieren. Da davon ausgegangen werden kann, dass ohnehin in vielen Fällen nicht der Abgeordnete selbst, sondern einer seiner Mitarbeiter für die Pflege des Internetangebots zuständig ist, erscheint deren Einbeziehung in die Befragung sinnvoll. Tatsächlich wurden 43 Prozent der Fragebögen von Mitarbeitern beantwortet. ${ }^{2}$

\section{Operationalisierung der Einflussfaktoren im Untersuchungsmodell}

Ziel der Untersuchung ist es zu erklären, aus welchen Gründen die Abgeordneten bestimmte Elemente für die Ausgestaltung ihrer Website nutzen und andere nicht. Die Ausgestaltung der persönlichen Website ist somit die abhängige Variable der Untersuchung (Abbildung 1). Sie umfasst eine Vielzahl von inhaltlichen, formalen und gestalterischen Aspekten und Dimensionen, die nicht alle berücksichtigt werden können. Welche Ausgestaltungselemente im Rahmen dieser Untersuchung einbezogen wurden, wird

\footnotetext{
Es ist zu vermuten, dass die Fragebögen auch bei anderen Politikerbefragungen tatsächlich von anderen Personen beantwortet werden, dies aber nicht offengelegt wird. Die einzelnen Fragen im Fragebogen wurden jeweils so formuliert, dass deutlich wurde, dass bei der Beantwortung jeweils die Sichtweise des Abgeordneten eingenommen werden sollte.
} 
im nächsten Abschnitt genauer erläutert. Im Folgenden werden zunächst die unabhängigen Faktoren des Modells betrachtet:

Abbildung 1: Untersuchungsmodell

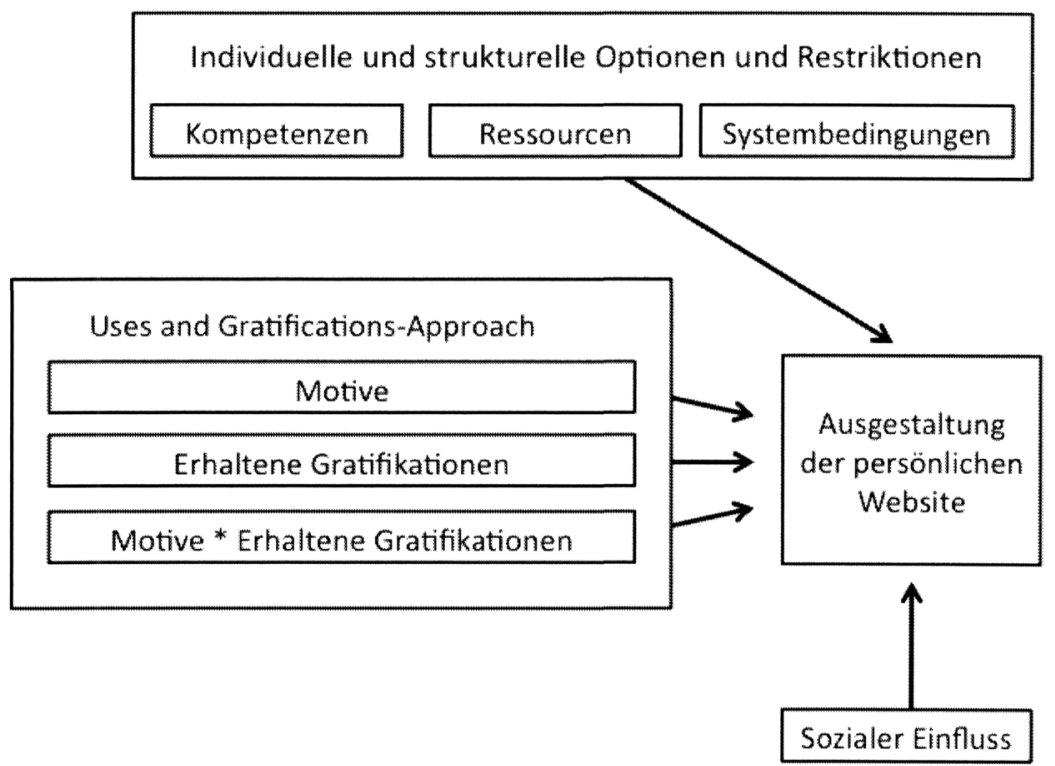

\section{Motive und erbaltene Gratifikationen}

Die Motive und erhaltenen Gratifikationen wurden mit umfangreichen Itembatterien gemessen. Es wurde jeweils die Zustimmung zu den Items auf einer 5-Punkte-Skala erhoben. ${ }^{3}$ Im ersten Teil des Fragebogens wurden die Zielsetzungen (Motive) ermittelt, die mit der Internetpräsenz erreicht werden sollen. Im letzten Teil wurde zusätzlich erfragt, inwieweit diese Ziele mit der Website tatsächlich erreicht werden. Beide Itembatterien wurden jeweils einer Faktorenanalyse unterzogen. Nachdem Items mit hoher Doppelladung entfernt worden waren, konnten jeweils drei Dimensionen ermittelt werden, die ähnliche Aspekte operationalisieren: Selbstdarstellung, Unterstützung und Feedback. Auf jede der drei Dimensionen laden jeweils zwei Items (Tabelle 1).

31 (= lehne stark ab) bis 5 (= stimme stark $\mathrm{zu}$ ) 
Tabelle 1: Motive und erbaltene Gratifikationen

\begin{tabular}{|c|c|c|c|c|}
\hline & \multicolumn{2}{|l|}{ Motive } & \multicolumn{2}{|l|}{ Erhaltene Gratifikationen } \\
\hline & $\begin{array}{l}\text { Ich möchte mit meiner } \\
\text { Internetpäsenz ... }\end{array}$ & $\begin{array}{l}\text { Mittel- } \\
\text { wert }\end{array}$ & $\begin{array}{l}\text { Durch meine Internet- } \\
\text { präsenz ... }\end{array}$ & $\begin{array}{l}\text { Mittel- } \\
\text { wert }\end{array}$ \\
\hline \multirow{3}{*}{$\begin{array}{l}\text { Selbstdar- } \\
\text { stellung }\end{array}$} & $\begin{array}{l}\text {... ein positives Selbstbild } \\
\text { vermitteln. }\end{array}$ & 4,1 & $\begin{array}{l}\text {... kann ich ein positives } \\
\text { Selbstbild in der Öf- } \\
\text { fentlichkeit vermitteln. }\end{array}$ & 4,2 \\
\hline & $\begin{array}{l}\text {... über meine eigene Ar- } \\
\text { beit und Person in- } \\
\text { formieren. }\end{array}$ & 4,7 & $\begin{array}{l}\text {.. kann ich mich gut in } \\
\text { der Öffentlichkeit dar- } \\
\text { stellen. }\end{array}$ & 4,1 \\
\hline & Skalenmittelwert & 4,4 & Skalenmittelwert & 4,1 \\
\hline \multirow{3}{*}{$\begin{array}{l}\text { Unterstüt- } \\
\text { zung }\end{array}$} & $\begin{array}{l}\text {... Zustimmung für die } \\
\text { eigene politische Ar- } \\
\text { beit erhalten. }\end{array}$ & 3,9 & $\begin{array}{l}\text {... werde ich in meinem } \\
\text { Wahlkampf unterstützt. }\end{array}$ & 3,4 \\
\hline & $\begin{array}{l}\text {... Unterstützer für meine } \\
\text { politische Arbeit fin- } \\
\text { den. }\end{array}$ & 3,7 & $\begin{array}{l}\text { ‥ habe ich Unterstützer } \\
\text { für meine politische } \\
\text { Arbeit gefunden. }\end{array}$ & 3,0 \\
\hline & Skalenmittelwert & 3,8 & Skalenmittelwert & 3,2 \\
\hline \multirow{3}{*}{ Feedback } & $\begin{array}{l}\ldots \text { Feedback aus der Be- } \\
\text { völkerung erhalten. }\end{array}$ & 3,8 & $\begin{array}{l}\text {.. bekomme ich Feed- } \\
\text { back aus der Bevölke- } \\
\text { rung. }\end{array}$ & 3,3 \\
\hline & $\begin{array}{l}\text {... Möglichkeiten anbie- } \\
\text { ten, mit mir ins Ge- } \\
\text { spräch zu kommen. }\end{array}$ & 4,2 & $\begin{array}{l}\text {.. bekomme ich wichtige } \\
\text { Hinweise, die für mei- } \\
\text { ne politische Arbeit } \\
\text { hilfreich sind. }\end{array}$ & 3,4 \\
\hline & Skalenmittelwert & 4,0 & Skalenmittelwert & 3,4 \\
\hline
\end{tabular}

Am stärksten ausgeprägt ist das Motiv der Selbstdarstellung. Dieses Motiv ist aber nicht nur das wichtigste, sondern wird - zumindest in der Wahrnehmung der Abgeordneten - auch durch die Website am besten befriedigt. An zweiter Stelle folgt der Wunsch, von den Bürgern Feedback zu erhalten. Diese Zielsetzung wird nach Ansicht der Abgeordneten durch die Website nicht ganz so gut erfüllt. An dritter Stelle - aber immer noch mit sehr hohen Zustimmungswerten - folgt das Ziel, durch die persönliche Website politische Unterstützung zu gewinnen. Nach Maßgabe der Mittelwerte gelingt auch diese nicht ganz im erhofften Maße. Im Anschluss an das Wert-Erwartungsmodell (Esser, 1999; Rayburn/Palmgreen, 1984) wurde durch die Multiplikation von Motiven und erhaltenen Gratifikationen für jede der drei Dimensionen zusätzlich auch ein Interaktionsfaktor berechnet. Dieser Faktor hat besonders hohe Werte, wenn eine Zielsetzung sehr ausgeprägt ist und gleichzeitig durch das Webangebot als gut erreichbar 
Jens Wolling, Anja Schmolinsky, Martin Emmer

eingeschätzt wird. Besonders niedrige Werte werden erzielt, wenn ein Ziel weder angestrebt noch erreicht wird.

\section{Optionen und Restriktionen}

Die individuellen und strukturellen Optionen und Restriktionen setzen sich aus insgesamt vier Variablen zusammen. Auf der individuellen Ebene handelt es sich dabei um drei Faktoren: Die wahrgenommenen Restriktionen im a) Zeitbudget, b) im sonstigen Ressourcenbudget sowie c) die vorhandene Internetkompetenz bei den Abgeordneten und ihren Teams.

Die Restriktionen im Zeit- und Ressourcenbudget wurden jeweils mit zwei Items erhoben. Auch hier wurde wiederum die Zustimmung auf 5-Punkte-Skalen gemessen (Tabelle 2). Die Operationalisierung der Internetkompetenz war etwas komplizierter. Erhoben wurde die subjektive Kompetenzeinschätzung und zwar jeweils separat für den Abgeordneten selbst und für seine Mitarbeiter. Die Messung erfolgte ebenfalls auf einer 5-Punkte-Skala4. Des Weiteren wurde erfragt, wer für die Betreuung der Website zuständig ist. Je nachdem, ob der Abgeordnete selbst oder seine Mitarbeiter die Website betreuen, wurde die entsprechende Kompetenzmessung für die Indexbildung herangezogen. Wenn die Website durch eine externe Agentur gepflegt wird, ist davon auszugehen, dass diese über eine hohe Kompetenz verfügt und der Kompetenzindex entsprechend mit dem Höchstwert „fünf“ kodiert werden kann.

Tabelle 2: Restriktionen und Optionen auf der individuellen Ebene

\begin{tabular}{lc}
\hline & Mittelwert \\
\hline Zeitrestriktionen & 3,9 \\
Aufbau und Pflege einer eigenen Webseite sind sehr zeitintensiv. & 4,0 \\
Durch meine zahlreichen Verpflichtungen habe ich wenig Zeit. & 4,0 \\
Skalenmittelwert & 3,0 \\
\hline Ressourcenrestriktionen & 2,7 \\
Die mir zur Verfügung stehenden finanziellen Mittel sind sehr begrenzt. \\
$\quad$ Wenn mir mehr Ressourcen zur Verfügung stehen würden, würde ich sie \\
in meine Webseite investieren. & 2,9 \\
$\quad$ Skalenmittelwert & 4,4 \\
\hline Wie schätzen Sie Ihre eigenen Internetkenntnisse und die Ihrer Mitarbeiter ein? & \\
\hline
\end{tabular}

Die Mittelwerte zeigen, dass die Zeitrestriktionen von den Abgeordneten deutlich stärker wahrgenommen werden als Restriktionen durch andere knappe Ressourcen. Feh-

$4 \quad 1$ (= sehr gering) bis 5 (= sehr gut) 
lende Internetkompetenz scheint hingegen kaum ein Problem darzustellen. Mit einem Mittelwert von 4,4 wird die vorhandene Kompetenz im Durchschnitt besser als "gut“ beurteilt.

Um den Einfluss der strukturellen Restriktionen und Optionen zu erfassen, wurde im Anschluss an die Überlegungen von Zittel (2009) die Unterscheidung zwischen Direkt- und Listenkandidat in den Modellen berücksichtigt. 44 Prozent der Befragungsteilnehmer sind als Direkt- und 56 Prozent als Listenkandidaten in die Landtage eingezogen. $^{5}$

\section{Sozrale Einflïsse}

Der letzte Faktor, der im Modell Berücksichtigung finden soll, sind die sozialen Einflüsse. Um deren Bedeutung zu ermitteln, wurden die Landtagsabgeordneten gebeten, einzuschätzen, wie wichtig es aus Sicht anderer Gruppen ist, dass Abgeordnete eine eigene Website besitzen. Verwendet wurde auch hier eine 5-Punkte-Skala ${ }^{6}$. Insgesamt wurde die Wichtigkeit für sechs Gruppen abgefragt: Eigene Mitarbeiter (Mittelwert = 4,1), eigene Partei $(4,1)$, Wähler (3,9), eigene Fraktion (3,9), Journalisten $(3,8)$ und eigene Bekannte (3,3). Da eine Faktorenanalyse der Items eine Ein-Faktor-Lösung ergab und auch die Reliabilität der Skala aus diesen sechs Items sehr gut war (Alpha $=.84$ ) wurde ein Gesamtindex „Einstellung des sozialen Umfeldes“ (3,8) konstruiert.

Die Operationalisierung der abhängigen Variablen wird im Zusammenhang mit der Beantwortung der beiden deskriptiven Fragestellungen im Ergebnisteil dargestellt.

\section{Ergebnisse}

Die Ergebnisdarstellung gliedert sich in drei Teile: Im ersten Teil wird gezeigt, wie viele Abgeordnete über eine private Website verfügen und wie intensiv sie diese pflegen. In zweiten Teil soll untersucht werden, welche Bedeutung die verschiedenen Ausgestaltungsoptionen für die Abgeordneten jeweils haben. Im dritten Teil wird dann das oben entwickelte Erklärungsmodell geprüft und dargestellt, welchen empirisch nachweisbaren Einfluss die verschiedenen theoretisch bestimmten Faktoren auf die Ausgestaltung der Websites haben.

5 Ursache für das leichte Übergewicht an Listenkandidaten ist die Tatsache, dass das Wahlrecht einiger Bundesländer keine Direktmandate vorsieht und alle Abgeordneten über Listenwahl bestellt.

$6 \quad 1$ (= eher unwichtig) bis 5 (= sehr wichtig) 
Frage 1: Welche Landespolitiker nutzen eine persönliche Website für ibre politische Kommunikation und wie intensiv pllegen sie diese?

Die Befragungsergebnisse zeigen, dass 91 Prozent der Landtagsabgeordneten, die an der Befragung teilgenommen haben, über eine eigene Website verfügen. Nur 9 Prozent nutzen diese Kommunikationsmöglichkeit nicht. Die Beteiligung an der Befragung ist somit unter den Websitebesitzern offenbar überproportional hoch ausgefallen, da nach Angabe von Altmeyer et al. (2007) die Ausstattung mit privaten Websites bei Landtagsabgeordneten im Jahr 2007 - nur etwa ein Jahr vor der vorliegenden Untersuchung erst bei rund 70 Prozent lag.

Die Entscheidung für oder gegen eine eigene Website fällt in den verschiedenen Abgeordnetengruppen insgesamt sehr ähnlich aus: Der Anteil der Abgeordneten ohne persönliche Website ist bei den Frauen nur geringfügig höher $(12 \%)$ als bei den Männern $(8 \%)$. Das gleiche gilt für die Listenkandidaten $(12 \%)$ im Vergleich zu den Direktkandidaten (6\%). Dieser geringe Unterschied verdeutlicht, dass die Entscheidung für oder gegen eine eigene Website von systemischen Einflüssen offenbar kaum geprägt wird. Etwas deutlicher fällt der Unterschied zwischen den Abgeordneten der drei kleinen Parteien (15\% ohne persönliche Website) und denen der beiden großen Parteien aus ( $8 \%$ ). Besonders viele Abgeordnete ohne eigene Internetpräsenz findet man in den kleinen Parlamenten (20\%). In den mittelgroßen ist der Anteil durchschnittlich $(9 \%)$, in den großen Parlamenten ist er am geringsten $(7 \%)$. Im Ost-West-Vergleich zeigt sich, dass der Anteil der Parlamentarier ohne eigene Webpräsenz in den ostdeutschen Parlamenten mit 12 Prozent etwas höher ausfällt als im Westen mit sieben Prozent. Überraschend ist sicherlich, dass die Abgeordneten der bürgerlichen Parteien CDU/CSU/FDP in der Stichprobe sogar noch etwas stärker im Netz vertreten sind (92\%) als die Abgeordneten von SPD, Grünen und den Linken (89\%). Unerwartet ist sicherlich auch der Befund, dass der Anteil derjenigen, die keine eigene Seite haben, unter den jüngeren Abgeordneten (bis 50 Jahre) etwas größer ist $(11 \%$ ) als unter den älteren $(7 \%)$.

Deutlichere Unterschiede zwischen den Abgeordneten mit und ohne eigene Website sind hingegen bezüglich der vermuteten Wichtigkeit für relevante Bezugsgruppen festzustellen. Diejenigen, die eine eigene Website betreiben, finden ein solches Angebot nicht nur persönlich wichtiger, sondern sie gehen auch stärker davon aus, dass andere Bezugsgruppen eine eigene Website bei einem Landtagsabgeordneten für wichtig halten (Tabelle 3). Besonders ausgeprägt sind die Unterschiede zwischen den Gruppen hinsichtlich der vermuteten Wichtigkeit für die eigenen Mitarbeiter und für die Wähler. Mit Ausnahme der vermuteten Wichtigkeit für die Fraktion sind die Unterschiede bei allen Bezugsgruppen signifikant. Während die Abgeordneten mit eigener Website sowohl bei internen als auch bei externen Bezugsgruppen von einer hohen Wichtigkeit 
ausgehen, gehen die Abgeordneten ohne eigene Website noch am ehesten bei den internen Akteuren der eigenen Partei und Fraktion von einer hohen Wichtigkeitszuschreibung aus. Bei externen Bezugsgruppen wie Journalisten, Wählern und persönlichen Bekannten rechnen sie weniger damit, dass diese ein solches Angebot wichtig finden. Insgesamt sprechen die Befunde dafür, dass die Abgeordneten sich dann für eine eigene Website entscheiden, wenn sie annehmen, dass ihre engsten Mitarbeiter und ihre Wähler dies von ihnen erwarten. Die vermutete Bedeutungszuschreibung durch Partei oder Fraktion scheint hingegen nicht so relevant zu sein.

Tabelle 3: Wichtigkeit der persönlichen Website aus der Sicht relevanter Bezugsgruppen

\begin{tabular}{lccc}
\hline $\begin{array}{l}\text { Wichtigkeit einer persönlichen Web- } \\
\text { site von Landtagsabgeordneten ... }\end{array}$ & $\begin{array}{c}\text { Abgeordnete mit } \\
\text { eigener Website } \\
\mathrm{n} \geq 123 \\
\text { Mittelwert }\end{array}$ & $\begin{array}{c}\text { Abgeordnete obne } \\
\text { eigene Website } \\
\mathrm{n} \geq 11 \\
\text { Mittelwert }\end{array}$ & $\begin{array}{c}\text { Signifikanz } \\
\text { (2-seitig) }\end{array}$ \\
\hline ... für den Abgeordneten selbst & 4,4 & 3,4 & $<.001$ \\
... für die eigenen Mitarbeiter & 4,2 & 3,2 & $<.01$ \\
... für die eigene Partei & 4,1 & 3,5 & $<.05$ \\
... für die eigenen Wähler & 4,0 & 3,3 & $<.01$ \\
... für die eigene Fraktion & 3,9 & 3,6 & $\mathrm{n}$. s. \\
... für Journalisten & 3,8 & 3,2 & $<.05$ \\
... für die eigenen Bekannten & 3,4 & 2,6 & $<.05$ \\
\hline
\end{tabular}

1 (= eher unwichtig) bis 5 (= sehr wichtig)

Die dargestellten Ergebnisse zeigen, dass die weitaus meisten Landtagsabgeordneten über eine eigene Website verfügen. Die Entscheidung für oder gegen eine Website ist nicht auf strukturelle oder soziodemographische Faktoren zurückzuführen.

Doch mit der Einrichtung einer Website ist erst der erste Schritt getan. Die Qualität eines Onlineangebots hängt ganz entscheidend von dessen Pflege ab. Insbesondere die Aktualisierungshäufigkeit ist ein wichtiger Qualitätsindikator, der auch in den meisten früheren Untersuchungen Beachtung gefunden hat. Deswegen ist die Aktualisierungshäufigkeit die erste abhängige Variable dieser Untersuchung. Die Bedeutung einer regelmäßigen Aktualisierung ihrer Website ist den meisten Abgeordneten bewusst, denn zwei Drittel von ihnen aktualisieren ihre Website mindestens ein Mal wöchentlich. Jeder Sechste macht dies sogar fast täglich. Auf der anderen Seite findet aber bei einem Drittel der Abgeordneten eine Aktualisierung nicht einmal wöchentlich statt. Fünf Prozent machen dies sogar seltener als ein Mal pro Monat. Auch der Zeitaufwand variiert beträchtlich: Ungefähr 60 Prozent widmen ihrem Angebot bis zu zwei Stunden Pflege 
wöchentlich. Weitere 30 Prozent investieren bis zu 4 Stunden und immerhin 10 Prozent nehmen sich noch mehr Zeit für die Pflege ihres Webangebots.

Frage 2: Für wie bedeutsam erachten die Abgeordneten die unterschiedlichen Ausgestaltungsoptionen für ibre persönliche Website?

Die zweite deskriptive Forschungsfrage, die im Rahmen der Untersuchung beantwortet werden soll, bezieht sich auf die Ausgestaltung der Website. Um diese Frage zu beantworten, wurde den Abgeordneten eine umfangreiche Liste mit strukturellen und inhaltlichen Angeboten vorgelegt und dann die Frage gestellt, ob sie diese Optionen auf ihrer Website einsetzen. Um nicht nur die Implementation der verschiedenen Kommunikationsangebote zu erfassen - die ebenfalls durch eine Inhaltsanalyse hätten ermittelt werden können -, sondern um auch deren subjektive Bedeutung zu erheben, wurden die Abgeordneten befragt, für wie wichtig sie diese Angebote jeweils erachten. Die Antworten wurden einer Faktorenanalyse unterzogen (Tabelle 4). Insgesamt konnten sechs Einsatzbereiche ermittelt werden, in die die Ausgestaltungsmerkmale unterteilt werden können.

Im Anschluss an Emmer (2005) erfolgte eine Einteilung der Kommunikationsformen in drei Bereiche: Informationskommunikation, interpersonale Kommunikation und Partizipationskommunikation. Die Informationskommunikation gliedert sich wiederum in drei Teile: Dabei handelt es sich erstens um Informationen zur allgemeinen politischen Arbeit des Abgeordneten und seiner Partei, zweitens um persönliche Informationen über den Abgeordneten und drittens um Wahlkampfinformationen. Bei den interpersonalen Kommunikationsangeboten konnten zwei Dimensionen ermittelt werden. Zum einen sind dies Angebote, die den Bürgern die Möglichkeit eröffnen, mit dem Abgeordneten in einen individuellen interpersonalen Austausch einzutreten; zum anderen handelt es sich um Kommunikationsoptionen, um mit den Bürgern eine öffentliche Debatte zu führen. Bezüglich der Partizipationsangebote wurde nur eine Dimension ermittelt, die sowohl Angebote zur allgemeinen Parteiarbeit als auch wahlkampfbezogene Optionen umfasst.

Unabhängig von dieser Einteilung wurde auch die Relevanz von Serviceangeboten auf den Websites erhoben. Sie konnten den genannten sechs Dimensionen nicht sinnvoll zugeordnet werden und wurden deshalb in einem separaten Index zusammengefasst (Tabelle 5). 
Tabelle 4: Bedeutung von Angeboten zur Informations-, interpersonalen und Partizipationskommunikation

\begin{tabular}{|c|c|c|c|c|c|c|}
\hline & $\begin{array}{l}\text { Individu- } \\
\text { elle inter- } \\
\text { personale } \\
\text { Kommu- } \\
\text { nikation }\end{array}$ & $\begin{array}{l}\text { Politische } \\
\text { Themen- } \\
\text { Informa- } \\
\text { tionen }\end{array}$ & $\begin{array}{l}\text { Persönliche } \\
\text { Infor- } \\
\text { mationen }\end{array}$ & $\begin{array}{l}\text { Wahlkampf- } \\
\text { informa- } \\
\text { tionen }\end{array}$ & $\begin{array}{l}\text { Partizi- } \\
\text { pation }\end{array}$ & $\begin{array}{l}\text { Öffentliche } \\
\text { inter- } \\
\text { personale } \\
\text { Kommuni- } \\
\text { kation }\end{array}$ \\
\hline $\begin{array}{l}\text { Kontakt analog: } \\
\text { Telefon, Adresse, ... }\end{array}$ & .93 & & & & & \\
\hline $\begin{array}{l}\text { Kontakt digital: } \\
\text { E-Mail, Kontakt- } \\
\text { formular ... }\end{array}$ & .92 & & & & & \\
\hline $\begin{array}{l}\text { Positionen zu politi- } \\
\text { schen Streitfragen }\end{array}$ & & .84 & & & & \\
\hline $\begin{array}{l}\text { Informationen zu } \\
\text { aktuellen Themen }\end{array}$ & & .74 & & & & \\
\hline $\begin{array}{l}\text { Reden, Aufsätze, } \\
\text { Presseerklärungen }\end{array}$ & & .68 & & & & \\
\hline $\begin{array}{l}\text { Persönliche } \\
\text { Informationen }\end{array}$ & & & .93 & & & \\
\hline $\begin{array}{l}\text { Informationen über } \\
\text { das Wahlkampfteam }\end{array}$ & & & & .86 & & \\
\hline $\begin{array}{l}\text { Informationen zum } \\
\text { Wahlsystem }\end{array}$ & & & & .66 & & \\
\hline $\begin{array}{l}\text { Informationen über } \\
\text { Wahlkreis }\end{array}$ & & & & .63 & & \\
\hline $\begin{array}{l}\text { Wahlkampf-Online- } \\
\text { Spende (Konto) }\end{array}$ & & & & & .84 & \\
\hline $\begin{array}{l}\text { Parteibeitritt online } \\
\text { (Formular) }\end{array}$ & & & & & .74 & \\
\hline $\begin{array}{l}\text { Wahlkampftätigkeit } \\
\text { (Kontaktformular) }\end{array}$ & & & & & .68 & \\
\hline Forum & & & & & & .89 \\
\hline Gästebuch & & & & & & .89 \\
\hline Nutzer-Befragung & & & & & & .75 \\
\hline Eigenwerte & 1,4 & 1,4 & .09 & 0,9 & 2,2 & 4,6 \\
\hline Skalenreliabilität Alpha & .86 & .64 & - & .64 & .83 & .89 \\
\hline Skalenmittelwert & 4,4 & 3,7 & 3,7 & 1,7 & 1,0 & 0,8 \\
\hline
\end{tabular}

Hauptkomponentenanalyse mit Varimax-Rotation (Vorgabe: 6 Faktoren), $76 \%$ erklärte Varianz, alle Faktorladungen $>.45, \mathrm{KMO}=.73$ 
Betrachtet man die Mittelwerte der sieben erstellten Indizes, dann zeigt sich, dass die befragten Abgeordneten den Möglichkeiten zur Aufnahme individueller interpersonaler Kommunikation mit den Bürgern die höchste Bedeutung zumessen. An zweiter und dritter Stelle folgen gleichauf die allgemeinen politischen Informationsangebote über die Arbeit der Partei und der Abgeordneten sowie die privaten Informationen. Eine bereits deutlich geringere Wichtigkeit messen die Befragten den Serviceangeboten und den wahlkampfbezogenen Informationen bei. Deutlich abgeschlagen am Ende der Wichtigkeitsskala finden sich die Partizipationsangebote sowie die Möglichkeiten zur öffentlichen interpersonalen Kommunikation.

Tabelle 5: Bedeutung von Serviceangeboten

\begin{tabular}{lc}
\hline & Item-Mittelwerte \\
\hline Newsletter bzw. mobiler Newsletter & 1,8 \\
Online-Bestellmöglichkeiten: Plakate, Flugblätter, Broschüren & 1,0 \\
Linksammlung & 3,2 \\
Veranstaltungskalender: Termine öffentlicher Auftritte des Abgeordneten & 3,3 \\
Skalenmittelwert & 2,3 \\
\hline Skalenreliabilität Alpha & .66 \\
\hline
\end{tabular}

Durch welche Faktoren werden die Entscheidungen für spezifische Ausgestaltungsoptionen der persönlichen Website beeinflusst?

Die entscheidende Zielsetzung dieser Untersuchung ist die Ermittlung relevanter Erklärungsfaktoren für die Pflege und Ausgestaltung der Website. Insgesamt acht abhängige Variablen wurden in den beiden vorhergehenden Abschnitten ermittelt: Neben der Aktualisierungshäufigkeit handelt es sich dabei um drei Indikatoren der Informationskommunikation, um zwei der interpersonalen Kommunikation sowie um je einen Indikator für die Nutzung von Partizipations- und Serviceangeboten.

Zur Erklärung wurden - wie oben bereits dargestellt - Faktoren aus drei Bereichen herangezogen. Dabei handelt es sich erstens um motivationale Erklärungsansätze aus der Uses-and-Gratifications-Forschungstradition: Motive, erhaltene Gratifikationen und die aus beiden Elementen zusammengesetzten Interaktionsfaktoren (insgesamt neun Variablen). Zweitens werden Restriktionen und Optionen (insgesamt vier Variablen) sowie drittens ein Indikator für die sozialen Einflüsse durch relevante Bezugsgruppen untersucht. 
Um die Bedeutung der genannten Faktoren zu ermitteln, wurden Regressionsanalysen ${ }^{7}$ durchgeführt. Die Modelle wurden schrittweise entwickelt, indem nacheinander für jede Variable geprüft wurde, ob sie einen signifikanten ${ }^{8}$ Beitrag zur Erklärung der jeweils abhängigen Variablen leisten kann. Nur Variablen, für die ein signifikanter Einfluss ermittelt wurde, wurden in die endgültigen Modelle aufgenommen. Diese Modelle sind in den Tabellen 6 und 7 dargestellt. Die erklärte Varianz $\left(\mathrm{R}^{2}\right)$ beträgt zwischen 13 und 26 Prozent. Dies kann als durchaus zufriedenstellend bezeichnet werden, zumal die Erklärungskraft jeweils mit relativ wenigen Variablen (maximal drei) erreicht wird.

Tabelle 6: Erklärungsmodelle für Aktualisierungshäufigkeit und Informationsangebote

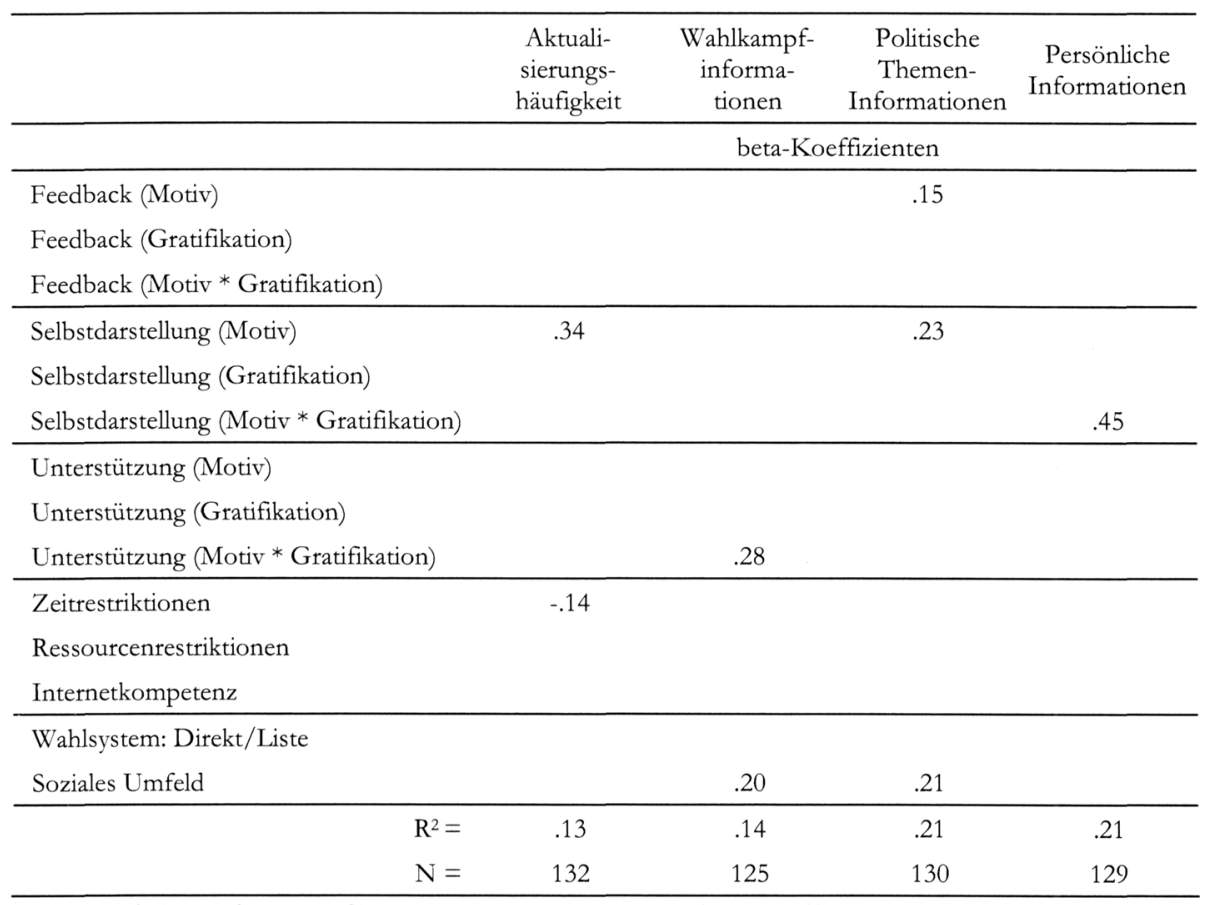

In den Modellen sind nur signifikante $(\mathrm{p}<.05$, einseitiger Test) beta-Koeffizienten enthalten.

Regressionsberechnungen unter paarweisem Ausschluss fehlender Werte.

8 Es wurden einseitige Signifikanztests eingesetzt, was zur Folge hat, dass Zusammenhänge in der erwarteten Richtung bereits bei geringeren Effekten bzw. kleineren Fallzahlen signifikant werden als im zweiseitigen Test. Einseitige Tests erscheinen hier angemessen, da zwar keine gerichteten Hypothesen formuliert wurden, jedoch grundsätzlich davon ausgegangen werden kann, dass die motivationalen Faktoren einen positiven Einfluss haben. Das gleiche gilt für die Internetkompetenz, das Wahlsystem und den sozialen Einfluss. Negative Zusammenhänge werden mit den beiden Restriktionen erwartet. 
In sieben von acht Modellen kann ein Einfluss motivationaler Faktoren nachgewiesen werden. Welche Dimensionen sich dabei jeweils als erklärungskräftig erweisen, variiert erheblich. Die ermittelten Unterschiede zwischen den Modellen lassen sich aber inhaltlich plausibel erklären.

Das Selbstdarstellungsmotiv hat einen positiven Einfluss auf die Aktualisierungshäufigkeit sowie auf die Informationskommunikation und zwar speziell auf das Angebot von persönlichen und politischen Informationen, wobei es die Nutzung persönlicher Informationen vor allem dann fördert, wenn die Abgeordneten das Gefühl haben, dass es ihnen durch das Angebot wirklich gelingt, sich positiv darzustellen. Einen leicht positiven Effekt hat das Selbstdarstellungsmotiv auch auf den Einsatz individueller interpersonaler Kommunikationsangebote: Die Ergebnisse zeigen, dass die Abgeordneten eine positive Selbstdarstellung vor allem durch die Bereitstellung von entsprechenden Informationen zu erreichen versuchen. Hingegen kann beispielsweise das Angebot von Partizipationsmöglichkeiten oder öffentliche interpersonale Kommunikation nach Einschätzung der Befragten nicht in bedeutsamer Weise zur Vermittlung eines positiven Selbstbildes beitragen.

Während sich bei der Selbstdarstellungsdimension durchgängig die Motivkomponente als bedeutsam erweist, sind es bei der Unterstützungsdimension die Gratifikationen. Der Einsatz von insgesamt drei Angebotsformen wird durch den Erhalt von entsprechenden Gratifikationen beeinflusst. Der Einsatz von Serviceleistungen wird durch wahrgenommene Unterstützungsgratifikationen beeinflusst, während die Bereitstellung von Wahlkampfinformationen und Partizipationsangeboten durch das Zusammenspiel von Motiven und Gratifikationen beeinflusst wird. Auch diese Befunde erscheinen plausibel. In allen drei Fällen sind die Angebote darauf ausgelegt, verschiedene Formen der Unterstützung zu generieren. Sowohl die Serviceangebote als auch die Wahlkampfinformationen und die Partizipationsangebote zielen darauf $a b$, Unterstützung für den Kandidaten und seine Partei zu fördern und zwar sowohl im Wahlkampf wie auch im Rahmen der politischen Alltagsarbeit.

Ebenso erweist sich die dritte Motivationsdimension - das Feedback - in drei Fällen als signifikanter Prädiktor. Hier sind es wiederum die Motive und nicht der Gratifikationen, die eine größere Rolle spielen. Einen besonders starken Einfluss hat das Feedbackmotiv auf die Bereitstellung von Angeboten zur individuellen interpersonalen Kommunikation. Daneben gibt es einen starken Effekt auf die Bereitstellung von Serviceangeboten und einen kleineren auf das Angebot politischer Themeninformationen. Insbesondere in den ersten beiden Fällen ist der Zusammenhang zwischen Feedbackmotiv und Kommunikationsangebot plausibel und nachvollziehbar: Die individuellen Kommunikationsangebote, aber auch die Serviceangebote zielen offensichtlich darauf ab, Feedback von den Bürgern zu erhalten. 
Der motivationale Erklärungsansatz hat sich somit in fast allen Modellen bewährt. Im Hinblick auf die Rolle von Optionen und Restriktionen fallen die Befunde differenzierter aus: Ein vergleichender Blick auf die beiden Tabellen zeigt, dass die Bereitstellung von Informationsangeboten nicht durch diesen Variablenkomplex beeinflusst wird, während sich bei allen anderen abhängigen Variablen die Optionen und Restriktionen als wirksam erweisen. Eine hohe Internetkompetenz wirkt sich positiv auf die Nutzung von Partizipations- und Serviceangeboten aus sowie auf die Bereitstellung von Möglichkeiten zur individuellen interpersonalen Kommunikation. Zeitrestriktionen haben hingegen einen negativen Effekt auf die Aktualisierungshäufigkeit.

Tabelle 7: Erklärungsmodelle für Interaktions-, Partizipations- und Serviceangebote

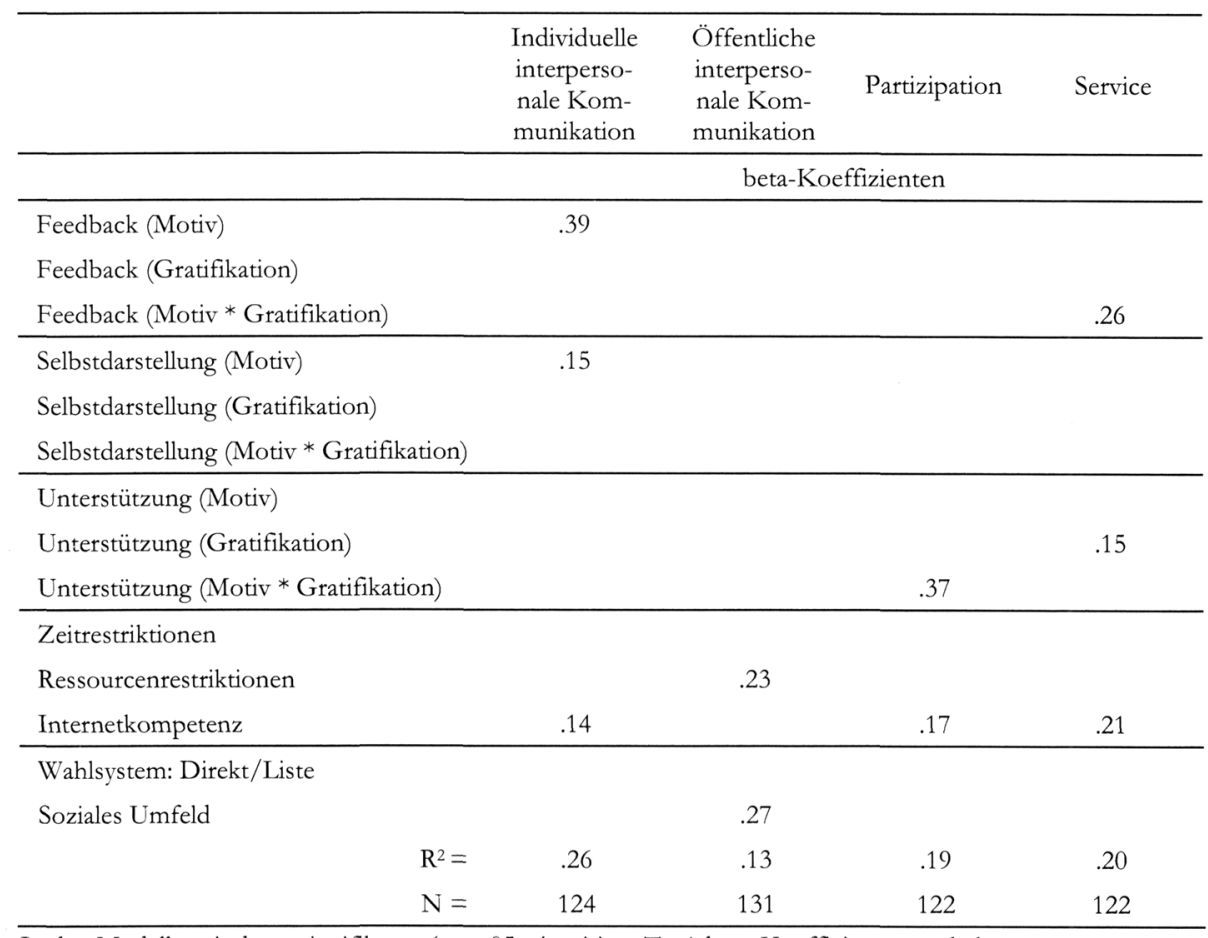

In den Modellen sind nur signifikante $(\mathrm{p}<.05$, einseitiger Test) beta-Koeffizienten enthalten.

Alle zuvor präsentierten Zusammenhänge entsprechen in ihrer Richtung den theoretischen Erwartungen. Der variierende Einfluss der verschiedenen Komponenten ist zudem in den allermeisten Fällen inhaltlich gut nachvollziehbar. Anders verhält es sich zumindest auf den ersten Blick - mit dem positiven Einfluss von Ressourcenrestriktionen auf den Einsatz von Instrumenten der öffentlichen interpersonalen Kommunikati- 
on. Je beschränkter die finanziellen Ressourcen wahrgenommen werden, desto häufiger werden diese Kommunikationsoptionen eingesetzt. Eine mögliche Erklärung für diesen Zusammenhang könnte sein, dass die Bereitstellung solcher Kommunikationsmöglichkeiten auf der Website zwar durchaus mit Kosten verbunden ist, diese Kosten aber im Vergleich zu alternativen Formen des öffentlichen Austauschs außerhalb des Internets überschaubar sind und deswegen im Fall kleinerer Budgets bevorzugt die Onlinevariante gewählt wird.

Anders als erwartet ist das Wahlsystem für die Nutzungsentscheidungen der Abgeordneten irrelevant. In keinem der acht Modelle wurde ein signifikanter Effekt ermittelt.

Die letzte Erklärungsdimension des Modells ist die Einstellung des sozialen Umfeldes. In drei Modellen zeigt sich ein positiver Effekt dieses Faktors. Wenn die Abgeordneten vermuten, dass die genannten Gruppen private Politikerwebsites für wichtig halten, dann fördert dies die Bereitstellung von Wahlkampf- und Themen-Informationen sowie die Einrichtung von Angeboten zur öffentlichen interpersonalen Kommunikation.

\section{Zusammenfassung und Ausblick}

Ein Abgeordneter sollte eine eigene Interpräsenz haben - darüber sind sich die befragten Landtagsmitglieder weitgehend einig. Einig sind sie sich zudem in der Annahme, dass auch die meisten der für sie relevanten Bezugsgruppen meinen, dass ein Abgeordneter eine private Website haben sollte. Angesichts dieser Befunde kann es nicht überraschen, dass die große Mehrheit der Landtagsabgeordneten tatsächlich über eine eigene Website verfügt. Die Entscheidung für eine eigene Internetpräsenz ist dabei weitgehend unabhängig von individuellen Merkmalen, politischer Zugehörigkeit oder regionaler Herkunft. Einzig die wahrgenommenen Einstellungen des sozialen Umfeldes spielen eine nennenswerte Rolle.

Die Entscheidung, ob eine Website notwendig ist, steht also bei den Landtagsabgeordneten nicht mehr zur Debatte. Nicht selbstverständlich ist hingegen, welche Inhalte und welche Kommunikationsmöglichkeiten auf der Website angeboten werden sollten. Die vorliegenden Inhaltsanalysen politischer Webangebote von Regierungen, Parteien und Politikern zeigen übereinstimmend, dass auf diesen Seiten das Potenzial des Internets nur ansatzweise ausgeschöpft wird (siehe dazu auch den Beitrag von Baumgartner/Zogg in diesem Band). Dieser Befund wird auch durch die hier vorgestellten Befragungsergebnisse bestätigt. Aufwändigere Formen der Interaktion und Partizipation werden von den Abgeordneten nicht für wichtig gehalten und kaum genutzt. Dieser Verzicht ist nur in geringem Maße auf fehlende Kompetenzen oder Restriktionen zu- 
rückzuführen. Die entscheidenden Einflussfaktoren sind in fast allen Fällen motivationaler Natur. Das bedeutet, dass die tatsächlich vorhandenen Webangebote, die weniger auf die Einbindung der Bürger und die politische Debatte setzen, sondern vielmehr auf politische Selbstdarstellung und die Generierung politischer Unterstützung abzielen, aus der Sicht der meisten Abgeordneten ihren Zweck damit hinreichend erfüllen.

Die große Bedeutung des Feedbackmotivs steht damit in einem deutlichen Widerspruch zur tatsächlichen Nutzung weitergehender interaktiver Kommunikationsformen. Die vorhandenen Unterschiede im Feedbackmotiv erklären zwar die Unterschiede in der Wichtigkeit der individuellen interpersonalen Kommunikationsangebote, zu einer verstärkten Nutzung öffentlicher interpersonaler Kommunikationsangebote tragen sie jedoch nicht bei. Die große Wertschätzung des Bürgerfeedbacks korrespondiert nicht mit einer entsprechenden Nutzung der Möglichkeiten. Vermutlich ist dies aber eine nicht nur rationale, sondern auch eine realistische Einschätzung der tatsächlichen Bedürfnisse des weitaus größten Teils des Elektorats.

Wie sich die Nutzung des Webs durch die Abgeordneten weiterentwickeln wird, hängt vermutlich entscheidend von der gesellschaftlichen Dynamik ab. Die Bedeutung sozialer Einflüsse - die Vermutung, dass relevante Bezugsgruppen bestimmte Erwartungen an die Handlungsweisen der Politiker haben - war nicht nur für die Einrichtung der Websites von Bedeutung, sondern auch für deren Ausgestaltung. Gerade bei den öffentlichen Formen der interpersonalen Kommunikation erweist sich dieser Faktor als bedeutsam. Es ist also zu erwarten, dass, wenn die Bürger diese Form des politischen Austauschs einfordern, die Abgeordneten mit großer Wahrscheinlichkeit mit der Einrichtung entsprechender Angebote reagieren werden. Wenn sie erwarten, dass es sich lohnt oder für eine Wiederwahl sogar notwendig ist, werden sie den partizipativen und interaktiven Elementen vermutlich mehr Aufmerksamkeit schenken als dies bisher der Fall ist.

\section{Literatur}

Altmeyer, Johannes/Böthling, Holger/Hübner, Wendelin/Schramm, Rüdiger et al. (2007): Der große p\&k Webseitentest. Welcher Politiker hat die beste Internetpräsenz? In: Politik \& Kommunikation, 49, 12 15.

Beck, Klaus (2006): Computervermittelte Kommunikation im Internet. München: Oldenbourg.

Döring, Nicola (2003): Politiker-Homepages zwischen Politiker-PR und Bürgerpartizipation. In: Publizistik, $48(1), 25-46$.

Druckman, James N./Kifer, Martin J./Parkin, Michael (2007): The Technological Development of Congressional Candidate Web Sites: How and Why Candidates Use Web Innovations. In: Social Science Computer Review, 25(4), 425-442. Online im Internet:

http://ssc.sagepub.com/cgi/content/abstract/25/4/425.

eCandidates 2002 (2002). Berlin: politik-digital.de u. a. Online im Internet: http://www.3-point.de/downloads/ecandidates2002.pdf.

Eisenführ, Franz/Weber, Martin (2003): Rationales Entscheiden. 4. Aufl. Berlin: Springer. 
Emmer, Martin (2005): Politische Mobilisierung durch das Internet? Eine kommunikationswissenschaftliche Untersuchung zur Wirkung eines neuen Mediums. München: Reinhard Fischer.

Emmer, Martin/Wolling, Jens (2009): „Online Citizenship“? Die Entwicklung der individuellen politischen Beteiligung im Internet. In: Bertelsmann Stiftung (Hrsg.): Lernen von Obama? Das Internet als Ressource und Risiko von Poliitk. Gütersloh: Verlag Bertelsmann Stiftung, 83-116.

Esser, Hartmut (1999): Soziologie: Spezielle Grundlagen. Band 1: Situationslogik und Handeln. Frankfurt am Main, New York: Campus.

Faas, Thorsten (2003): Landtagsabgeordnete in den Weiten des Netzes. Ergebnisse von Umfragen unter Landtagsabgeordneten zur Bedeutung des Internets in Politik und Wahlkämpfen. In: Rogg, Arne (Hrsg.): Wie das Internet die Politik verändert. Einsatzmöglichkeiten und Auswirkungen. Opladen: Westdeutscher Verlag, 55-65.

Fengler, Susanne/Ruß-Mohl, Stephan (2005): Der Journalist als „Homo oeconomicus“. Konstanz: UVK.

Kleinsteuber, Hans J./Fries, Meike (2003): Abgeordnete des Deutschen Bundestags und das Internet: Eine Umfrage im europäischen Kontext. Bericht von einer Baustelle. In: Rogg, Arne (Hrsg.): Wie das Internet die Politik verändert. Einsatzmöglichkeiten und Auswirkungen. 2003 Opladen: Westdeutscher Verlag, 81-95.

Koch-Mehrin, Silvana (2006): Wichtiger Informationskanal. In: Das Parlament, 24.04./02.05.2006. 13.

Kühnel, Steffen (2001): Kommt es auf die Stimme an? Determinanten von Teilnahme und Nichtteilnahme an politischen Wahlen. In: Koch, Achim/Wasmer, Martina/Schmidt, Peter (Hrsg.): Politische Partizipation in der Bundesrepublik Deutschland. Empirische Befunde und theoretische Erklärungen. Opladen: Leske + Budrich, 11-42.

Marx, Dorothea (2009): Landtagsabgeordnete im Fokus der Medien. Ihre Sicht auf Entstehung, Wirkung und Qualität landespolitischer Berichtserstattung. Baden-Baden: Nomos.

Noelle-Neumann, Elisabeth (1982): Die Schweigespirale: Öffentliche Meinung - unsere soziale Haut. München: Ullstein.

Palmgreen, Philip/Wenner, Lawrence A./Rayburn, J. D. II (1980): Relations between Gratifications Sought and Obtained. A Study of Television News. In: Communication Research, 7(2), 161-192.

Papacharissi, Zizi/Rubin, Alan M. (2000): Predictors of Internet Use. In: Journal of Broadcasting \& Electronic Media, 44(2), 175-196.

Paschen, Frank/Wieboldt, Sven (2001): Frühlingserwachen? Bundestagsabgeordnete im Test. politikdigital.de. Online im Internet: http://www2.politik-digital.de/test/mdbtest2001/spring.shtml.

Rayburn, J. D. II/Palmgreen, Philip (1984): Merging Uses and Gratifications and Expectancy-Value Theory. In: Communication Research, 11(4), 537-562.

Rolke, Lothar/Metz, Volker (2006): Parlamentarier-PR: Die Bedeutung von Presse- und Öffentlichkeitsarbeit für die Abgeordneten des Deutschen Bundestages 2006. Ergebnisse einer Intensivbefragung von 119 MdBs. Berlin: Helios.

Rössler, Patrick (1998): Wirkungsmodelle: die digitale Herausforderung. In: Rössler, Patrick (Hrsg.): OnlineKommunikation: Beiträge zur Nutzung und Wirkung. Opladen, Wiesbaden: Westdeutscher Verlag, $17-$ 46.

Schweiger, Wolfgang (2007): Theorien der Mediennutzung: Eine Einführung. Wiesbaden: VS Verlag.

Schweitzer, Eva J. (2005): Election Campaigning Online: German Party Websites in the 2002 National Elections. In: European Journal of Communication, 20(3), 327-351. Online im Internet: http://ejc.sagepub.com/cgi/content/abstract/20/3/327.

Shahla, Hossein (1999): Empirische Wahlforschung und Wählerrationalität: Zum Stellenwert der Sachthemenorientierung im Prozeß der Wahlentscheidung. Frankfurt am Main, Berlin: Lang.

Siebel, Michael (2006): Demokratische Offenheit. In: Das Parlament. 24.04./02.05.2006. 9.

Thurner, Paul W./Roth, Dieter (2001): Wählen als rationale Entscheidung. Die Modellierung von Politikreaktionen im Mehrparteiensystem. In: Zeitschrift für Parlamentsfragen, 32(1), 214-215.

Vowe, Gerhard/Wolling, Jens (2002): Wollen, Können, Wissen: Was erklärt die Unterschiede in der Internetnutzung durch Studierende? Ein empirischer Theorietest. In: Schmidt, Siegfried J./Baum, Achim (Hrsg.): Fakten und Fiktionen. Über den Umgang mit Medienwirklichkeiten. Konstanz: UVK, 379-391.

Wenner, Lawrence A. (1982): Gratifications Sought and Obtained in Program Dependency. A Study of Network Evening News Programs and 60 Minutes. In: Communication Research, 9(4), 539-560. 
Zittel, Thomas (2003): Vernetzte politische Kommunikation: Elektronische Demokratie als amerikanischer Sonderweg? In: Esser, Frank/Pfetsch, Barbara (Hrsg.): Politische Kommunikation im internationalen Vergleich. Grundlagen, Anwendungen, Perspektiven. Wiesbaden: Westdeutscher Verlag, 259-280.

Zittel, Thomas (2005): Direkte Personalisierte Wählerkommunikation im WWW - Wunsch, Wirklichkeit und Perspektiven. Gutachten im Auftrag des Deutschen Bundestages. Mannheim: Büro für TechnikfolgenAbschätzung des Deutschen Bundestags (TAB).

Zittel, Thomas (2007): Demokratie und Internet. Mehr Responsivität durch neue digitale Medien? Habilitationsschrift, Universität Mannheim, Mannheim.

Zittel, Thomas (2009): Entmedialisierung durch Neue Digitale Medien? Direkte Wählerkommunikation im WWW aus der Sicht von Abgeordneten des Deutschen Bundestages. In: Marcinkowski, Frank/Pfetsch, Barbara (Hrsg.): Politik in der Mediendemokratie: Politische Vierteljahresschrift Sonderheft 42: Verlag für Sozialwissenschaften, 366-389. 